\title{
SNAPSHOT 2: SWIMMING IN THE INNER SOURCE: A WORKSHOP WITH NICOLÁS NÚÑEZ
}

\author{
Cassiano Sydow Quilici
}

In 2016, on the occasion of the 'Performance and Mindfulness: International Symposium' at the University of Huddersfield, I had the opportunity of participating in a workshop led by Nicolás Núñez. Even though the workshop lasted for just a few days, I quickly noted its depth and realized that it was the fruit of many years of research. Throughout the week, I was able to absorb precious procedures and principles, both on a personal level and in such a way that led me to consider new perspectives on my own work. I would like to underline three aspects that were particularly important.

First, there is the contextualization of the exercises within a 'cosmology' that defines different states and ways of being: the quotidian state, the hero state and divine state. The idea is that the training of the actor not only represents a set of strategies for creating a heightened experience of bodymind, but it is also a perspective for understanding the world beyond the everyday. When an exercise is situated within a broader field we are able to understand the horizon of the practice.

Secondly, the specific exercise of 'contemplative running' resulted in an interesting personal experience. Running here is a form of relaxing the body, strongly connected with the group, for a long period of time, in circles, allowing us to experience a change in the quality of energy, expressed in the form of running itself. In the beginning, my impression was that the whole group was galloping, based on an awareness of the contact with the ground and the energy of the earth. As I began using my arms, as if gliding, my energy was renewed and the image that appeared was that of a flock of birds moving through the sky, leading to a transformation of a dense sensation into a more subtle experience. The idea of human body as a complex structure that 
connects dense and subtle energies can also be found in the practice of tai chi chuan and other traditional practices.

Finally, my contact with Núñez and his work brought me greater clarity about the possibilities of dialogues between Eastern contemplative practices and the wealth of pre-Colombian indigenous cultures.He himself emphasized this connection, having worked with traditional forms of Tibetan dance, some aspects of which have been incorporated into his proposals. All of this strengthened in me the desire to develop a new approach to the question of 'mindfulness' that takes into consideration the context of Latin America and its singularities. I am extremely grateful to Nicolás Núñez and the University of Huddersfield for having this opportunity. 